\title{
Differences in immune contextures among different molecular subtypes of gastric cancer and their prognostic impact
}

\author{
Joo Yeon $\mathrm{Kim}^{1}$ · Woo Gyeong Kim ${ }^{1}$ Chae Hwa Kwon ${ }^{3}$ - Do Youn Park ${ }^{2,3}$
}

Received: 12 February 2019 / Accepted: 15 May 2019 / Published online: 31 May 2019

(c) The International Gastric Cancer Association and The Japanese Gastric Cancer Association 2019

\begin{abstract}
Background Gastric cancers have been recently classified in accordance with their molecular characteristics, thus demonstrating the complex nature of cancers and an association with the immune contexture within the tumor microenvironment. This study aimed to investigate the correlation between the molecular subtype and immune contexture of gastric cancers.

Methods The immune contexture, including the type, density, and location of tumor-infiltrating lymphocytes (TILs), of gastric cancer patients was examined and immune subtypes were classified based on it. In particular, PD-L1 expression on tumor cells and TILs and Foxp $3^{+}$TILs was assessed in accordance with molecular subtypes.

Results High levels of visual TIL estimates and Foxp $3^{+}$TILs were markedly associated with increased overall survival $(P=0.001, P<0.001$, separately). Immune subtypes were associated with tumor size, gross type, depth of invasion, lymph node metastatic status, lymphovascular invasion, perineural invasion, and microsatellite instability status. EBV-positive (C1) and MSI (C2) gastric cancers, considered subtypes with better prognosis, were significantly associated with high TIL levels $(P<0.05)$. In contrast, epithelial-mesenchymal transition (EMT, C3) gastric cancers with poor overall survival displayed low levels of Foxp3 ${ }^{+}$TILs. Type II tumors (low level of TILs/low PD-L1 expression) displayed a significant correlation with poor overall survival $(P=0.004)$ and accounted for the highest proportion in the aberrant p53-expressing $(C 4)$ gastric cancers. Conclusion The molecular subtype of gastric cancers is correlated with the immune subtype, including immune contexture and PD-L1 expression, within the tumor microenvironment.
\end{abstract}

Keywords Gastric cancer · Tumor microenvironment · Tumor-infiltrating lymphocytes · Survival

Electronic supplementary material The online version of this article (https://doi.org/10.1007/s10120-019-00974-4) contains supplementary material, which is available to authorized users.

Chae Hwa Kwon

chkwon@pusan.ac.kr

$\triangle$ Do Youn Park

pdy220@pusan.ac.kr

1 Department of Pathology, Haeundae Paik Hospital, College of Medicine, Inje University, Busan, South Korea

2 Department of Pathology, Pusan National University Hospital and Pusan National University School of Medicine, Busan, South Korea

3 BioMedical Research Institute, Pusan National University Hospital, 179, Gudeok-ro, Seo-gu, Busan 49241, South Korea

\section{Introduction}

Cancer is a morphologically and genetically complex disease with major pathognomonic hallmarks [1]. Recently, The Cancer Genome Atlas (TCGA) research network has profiled and analyzed gastric cancers and revealed DNA, RNA, protein, and epigenetic aberrations and classified gastric cancers on the basis of their molecular characteristics [2]. This molecular classification of gastric cancer is associated with distinct clinical and histological characteristics and delineates the key features of genetic alterations for putative targeted therapy [3, 4].

In addition to the understanding of intrinsic molecular alterations in cancer, the tumor microenvironment has received increasing attention in cancer biology. During tumor development and progression, tumor cells interact with various host cells such as infiltrating immune cells, including antigen-presenting cells, neutrophils, $\mathrm{T}$ cells, and 
B cells, and the tumor microenvironment is influenced by the host immune system [5].

To decrease immunogenicity, tumor cells activate the immune checkpoint pathway, which is critical for maintaining self-tolerance. Cytotoxic T-lymphocyte-associated antigen 4 (CTLA4) and programmed cell death protein 1 (PD1) are immune checkpoint receptors that inhibit the $\mathrm{T}$ cell response and have been extensively studied in the clinical context of immunotherapy [6]. Immune checkpoint inhibitors targeting the CTLA4 and PD1 pathways have shown remarkable clinical effects [7-9]. Most recently, antiPD-1 checkpoint immunotherapy with pembrolizumab was approved for patients with recurrent or metastatic PD-L1 ${ }^{+}$ gastric cancer. However, favorable results were obtained for a small subset [10-12].

Therefore, to administer effective immune-based cancer treatments to gastric cancer patients, comprehensive assessment of the immune contexture is essential. In this study, the immune contexture, including the type, density, and location of tumor-infiltrating lymphocytes (TILs), was examined in gastric cancer patients. In particular, the correlation between the immunologic and molecular subtypes of gastric cancers was evaluated on the basis ofPD-L1 expression and TIL levels. Ultimately, this study aimed to determine the difference in the tumor microenvironment in accordance with the molecular subtype of gastric cancer and to identify their potential therapeutic relevance.

\section{Materials and methods}

\section{Tumor samples and histopathologic evaluation}

In total, 578 gastric adenocarcinoma tissue samples from patients who underwent gastrectomy with lymph node dissection as first-line treatment at the Pusan National University Hospital (PNUH, Busan, Korea) between 2009 and 2010 were reviewed, of which 406 were used to construct the tissue microarray (TMA). Finally, 297 cases, including those of early gastric cancer with submucosal invasion and advanced gastric cancer, were included. Since we aimed to investigate the pathologic characteristics and immune contextures of the tumor center and its invasive margin, patients with early gastric cancer without submucosal invasion were excluded. All included patients completed follow-up evaluation until February 2016.

The clinicopathological data for each patient were obtained by reviewing the patient's electronic medical records and pathologic reports; these included patient age and sex, tumor location, size and gross type, Lauren and WHO histologic type, depth of invasion (pT stage), lymph node metastatic status ( $\mathrm{pN}$ stage), lymphovascular and perineural invasion, microsatellite instability (MSI) status, mucin phenotype, and HER2 status.

\section{TMA construction}

For TMA analysis, sections of formalin-fixed paraffinembedded tissues were prepared and stained with hematoxylin and eosin (H\&E). All available H\&E-stained slides were reviewed for appropriate tumor areas with the highest TIL population at the tumor center and invasive margin. From each tumor, two representative cores of 2.0-mm diameter were obtained using the TMA instrument and inserted in a grid pattern into a recipient block.

\section{Immunohistochemical analysis and Epstein-Barr virus in situ hybridization (EBV ISH)}

Four-micrometer-thick sections from each TMA were probed with anti-CD3 (1:400, Dako, Carpinteria, CA, USA), anti-CD8 (1.200, 4B11, Leica, Microsystems, Wetzlar, Germany), anti-Foxp3 (1:100, 236A/E7, Abcam, Cambridge, UK), anti-PD-L1 (1:200, E1L3N, Cell signaling Technology, Danvers, MA, USA), anti-MLH1 (1:150, ES05, Novocastra, Leica Biosystems, Newcastle Upon Tyne, UK), anti-E-cadherin (1:100, 36B5, Novocastra), and anti-p53 (1:400, DO-7, Novocastra) antibodies, using automatic immunohistochemical staining devices (Benchmark XT; Ventana Medical System, Tucson, AZ, USA and Bond-Max; Leica Microsystems, Wetzlar, Germany).

First, the study cohort was classified into five molecular subtypes (C-subtypes) in accordance with MLH-1, E-cadherin, and p53 mRNA and protein expression levels [4]. Aberrant MLH-1 expression was defined as complete loss of nuclear staining. Tumor cells with diffuse, completely obliterated, or upregulated p53 nuclear staining was considered to aberrantly express p53. Cells displaying complete loss of membranous or cytoplasmic staining were considered to aberrantly express E-cadherin.

To evaluate the immune contexture of each molecular subtype (C-subtypes), including visually estimated TILs, $\mathrm{CD}^{+}, \mathrm{CD}^{+}$, and Foxp3 ${ }^{+} \mathrm{T}$ cells, the absolute numbers of labeled TILs at the tumor center (TC) and invasive margin (IM) were determined. Visually estimated TILs were defined as the number of eyeball-measured lymphoid cells in the $\mathrm{H} \& \mathrm{E}$ staining. CD3 and CD8 were detected in the cytoplasm of the TILs, Foxp3, nucleus. Two representative areas with the highest cell density at high magnification $(400 \times)$ were counted, and the higher value was selected. Regarding TILs, infiltration levels were categorized as low and high based on the median number of immunoreactive immune cells and visually estimated immune cells in the TC and IM (Supplementary Fig. 1). For statistical analysis, total TIL levels were determined from the sum of the level in the TC and 
IM. Low TIL levels in both the TC and IM indicated low total TIL levels.

PD-L1 expression was evaluated separately for tumor cells and TILs at the TC and IM. Membrane staining was performed to detect PD-L1, using human placental tissue as a positive control. Staining intensity was graded using a 4-point scale as follows: 0 (no staining), 1 (weak staining), 2 (moderate staining), or 3 (strong staining) (Supplementary Fig. 2 and 3). The percentage of positive cells was assessed at $5 \%$ increments. PD-L1-positive staining included more than $5 \%$ of positive cells showing moderate and strong intensity [13]. PD-L1 expression levels on tumor cells and TILs were categorized as low and high upon positive staining of both the TC and IM. Tumor cells or TILs with negative PD-L1 staining in both the TC and IM were considered to display PD-L1 downregulation. The others (PD-L1 negative in the TC and PD-L1 positive in the IM; PD-L1 positive in TC and PD-L1 negative in the IM; PD-L1 positive in the TC and PD-L1 positive in the IM) were considered to display PD-L1 upregulation. Furthermore, tumors were categorized as high and low in accordance with their total PD-L1 status in tumor cells and TILs. PD-L1 downregulation in both tumor cells and TILs indicated a low PD-L1 status; the others were considered to have high PD-L1 status.

For survival analysis, the study cohort was segregated into four immune subtypes (I-subtypes) based on the TIL levels and PD-L1 expression in tumor cells, similar to Teng's classification [14]: type I, high level of TILs and high PD-L1 status (TILs H/PD-L1 H); type II, low level of TILs and low PD-L1 status (TILs L/PD-L1 L); type III, low level of TILs and high PD-L1 status (TILs L/PD-L1 H); type IV, high level of TILs and low PD-L1 status (TILs H/PD-L1 L). Herein, levels of visual TIL estimates were considered a representative value of TIL levels.

The presence of EBV in tumor cells was confirmed via chromogenic ISH with EBV-encoded small RNA (EBERISH), using an automatic staining device (BOND-MAX), with a Novocastra EBER probe for EBV (Leica Biosystems). Strong nuclear staining of the tumor cells rendered them EBV positive.

\section{Statistical analysis}

All statistical analyses were performed using SPSS 24.0 (IBM Corporation, New York, NY, USA). Immune contexture and PD-L1 expression were analyzed for differences among subtypes, using the $\chi^{2}$ test, Fisher's exact test and Kruskal-Wallis test. The correlation between different immune cell densities was analyzed using the Spearman rank correlation test. Differences in clinicopathologic characteristics based on PD-L1 status were analyzed using the Student's $t$ test, $\chi^{2}$ test, or Fisher's exact test. Survival curves were derived from Kaplan-Meier estimates, and the curves were compared using a log-rank test. For all tests, statistical significance was set at $P<0.05$.

\section{Results}

\section{Clinical impact of the immune contexture and PD-L1 expression on gastric cancer}

The association between the clinicopathological characteristics and immune contexture of TILs, including cell type $\left(\mathrm{CD}^{+}, \mathrm{CD}^{+}\right.$, and Foxp3 $\left.{ }^{+} \mathrm{TILs}\right)$, and density is summarized in Table 1. The high level of visual TIL estimates was significantly associated with various histopathologic features including younger age $(P=0.007)$ and location in the body $(P=0.005)$, non-excavated gross type $(P=0.014)$, lower pT $(P<0.000)$ and pN stages $(P=0.002)$, and the absence of lymphovascular and perineural invasion $(P=0.003$ and $P<0.000$, separately). Moreover, Foxp3 upregulation was significantly associated with large tumor size $(P=0.015)$, and low level of Foxp $3^{+}$TILs was statistically associated with high stage of pT $(P<0.001)$ and $\mathrm{pN}(P=0.015)$ and the presence of lymphovascular invasion $(P=0.050)$. Regarding the prognostic impact of immune cells on gastric cancer, high levels of visual TIL estimates were significantly associated with a better prognosis $(P=0.001$, Fig. 1a). Furthermore, Foxp3 upregulation was significantly associated with better treatment outcomes $(P<0.001$, Fig. 1d). However, a high level of $\mathrm{CD}^{+}$and $\mathrm{CD} 8^{+}$TILs were associated with Lauren classification $(P=0.005$ and $P=0.011$, respectively), perineural invasion $(P=0.017$ and $P=0.007$, respectively) and MSI-H ( $P=0.021$ and $P=0.023$, respectively), but not with $\mathrm{pT}$ and $\mathrm{pN}$ stages and prognosis $(P=0.185$ and $P=0.179$, respectively, Fig. $1 \mathrm{~b}$ and c).

The association between PD-L1 expression and the clinicopathological features is shown in Table 2. Among the histopathologic parameters, tumor size $(P=0.024)$, pT stage ( $P$ $=0.043)$, lymphovascular invasion $(P=0.029)$, and MSI $(P$ $=0.033)$ were significantly associated with PD-L1 expression on tumor cells . However, PD-L1 expression of TILs was not associated with $\mathrm{pT}$ and $\mathrm{pN}$ stages and lymphovascular invasion. Moreover, PD-L1 upregulation on tumor cells and TILs was slightly, but not significantly associated with improved overall survival $(P=0.133$ and $P=0.644$, respectively, Fig. 2).

The study cohort was segregated into four immune subtypes (I-subtypes) on the basis of TIL level and PD-L1 expression in tumor cells as mentioned in "Materials and methods": type I (TILs H/PD-L1 H), type II (TILs L/PD-L1 L), type III (TILs L/PD-L1 H), and type IV (TILs H/PD-L1 $\mathrm{L}$ ), and the association between I-subtypes and clinicopathological characteristics was analyzed. These subtypes were associated with tumor size $(P=0.013)$, gross type 


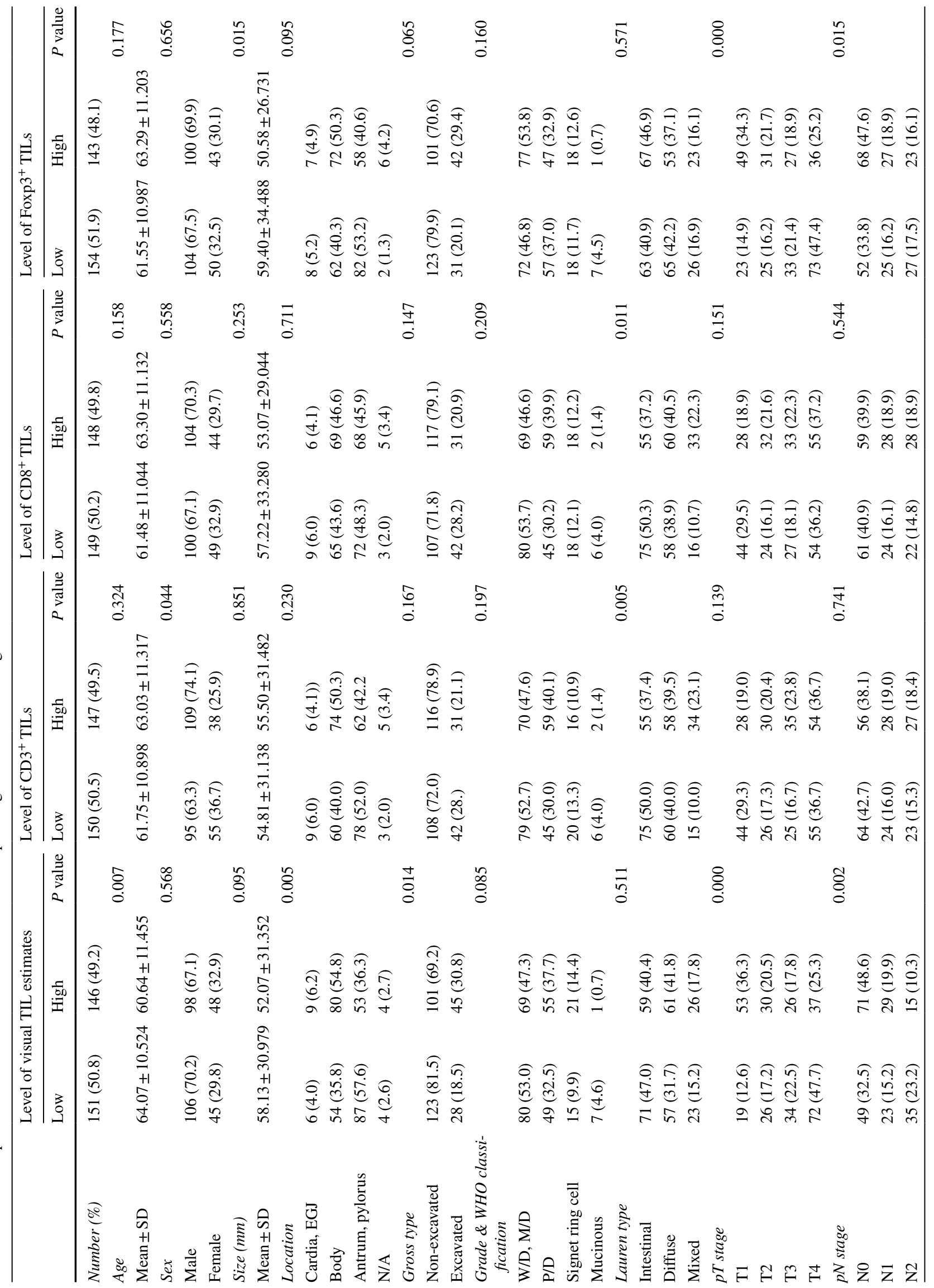




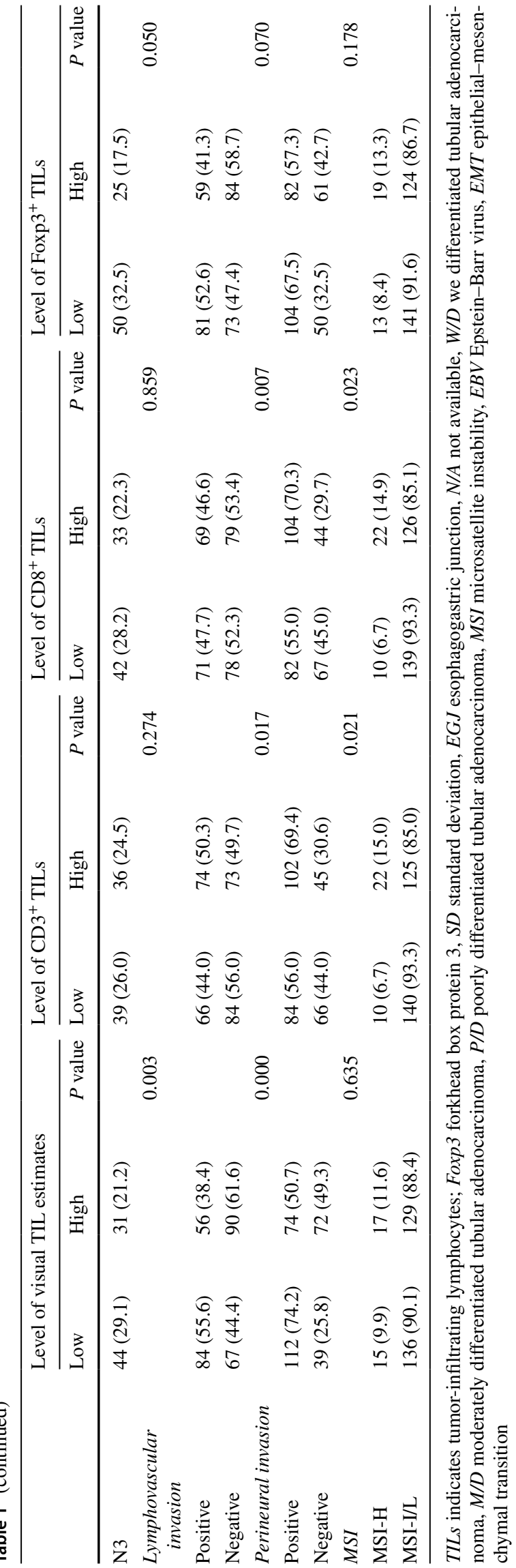

$(P=0.000)$, pT stage $(P=0.000)$, pN stage $(P<0.000)$, lymphovascular invasion $(P=0.000)$, perineural invasion $(P<0.000)$, and MSI status $(P<0.000)$ (Table 3$)$. Accordingly, type II tumors were significantly correlated with poor overall survival rate; type I, better overall survival rate; type III and IV, intermediate overall survival rate $(P=0.004$, Fig. 3a).

\section{Differences in immune contexture and PD-L1 expression among molecular subtypes and the association between immune and molecular subtypes within the tumor microenvironment}

According to a simple taxonomic sequence in our previous study [3], the cohort was divided into the following five molecular subtypes (C-subtype): C1 (EBV positive, $n=22$, $7.4 \%$ ), C2 (aberrant MLH-1 expression or MSI, $n=29$, 9.8\%), C3 (aberrant E-cadherin expression or EMT, $n=53$, $17.8 \%$ ), C4 (aberrant p53 expression, $n=119,40.1 \%$ ), and C5 (normal p53 expression, $n=74,24.9 \%$ ). These subtypes were associated with tumor size $(P=0.007)$, location $(P=0.049)$, grade, and WHO classification $(P<0.001)$, Lauren type $(P<0.001)$, lymph node metastasis (pN stage) $(P=0.008)$, perineural invasion $(P=0.004)$, and MSI status $(P<0.001)$ (Supplement Table 1$)$. Herein, the overall survival based on the $\mathrm{C}$-subtype was the same as that reported previously; MSI tumors revealed the best prognosis, followed by EBV-positive tumors, those with normal p53 expression, and those with aberrant p53 expression, with the $\mathrm{C} 3$ type showing the worst prognosis $(P<0.001)$ (Fig. 3b).

Differences in immune contexture and PD-L1 expression were evaluated on the basis of the molecular subtype (Table 4). Among them, $\mathrm{C} 1$ and $\mathrm{C} 2$, being subtypes with better prognosis, were significantly associated with high levels of immune density, including $\mathrm{CD}^{+}, \mathrm{CD} 8^{+}$, and Foxp $3^{+}$TILs, regardless of location $(P=0.001, P<0.001$, $P=0.005$ in TC, $P=0.018, P=0.001, P<0.001$ in IM, respectively). However, $\mathrm{C} 3$ type with poor overall survival displayed low level of Foxp $3^{+}$TILs compared with other C-subtype (32 cases (60.4\%) in TC, 36 cases (67.9\%) in IM). PD-L1 expression displayed high positivity rates in the $\mathrm{C} 1$ and $\mathrm{C} 2$ types at any location (TC and IM) and in any cell type (tumor cells or TILs). In multivariable analyses using Cox proportional hazards regression models, C3 subtype was identified as an independent poor prognostic factor compared with the other subtypes (hazard ratio, 2.105; $P=0.001$ ) (Supplement Table 2). Moreover, the proportion of subtypes $\mathrm{C} 1$ and $\mathrm{C} 2$ with a high total PD-L1 status was significantly high $(P<0.001$, Table 4$)$. The proportion of type I tumors was high in groups with a suitable prognosis, namely $\mathrm{C} 1$ and $\mathrm{C} 2$. The $\mathrm{C} 4$ subtype accounted for the greatest proportion of type II tumors (42.9\%, Table 4). 

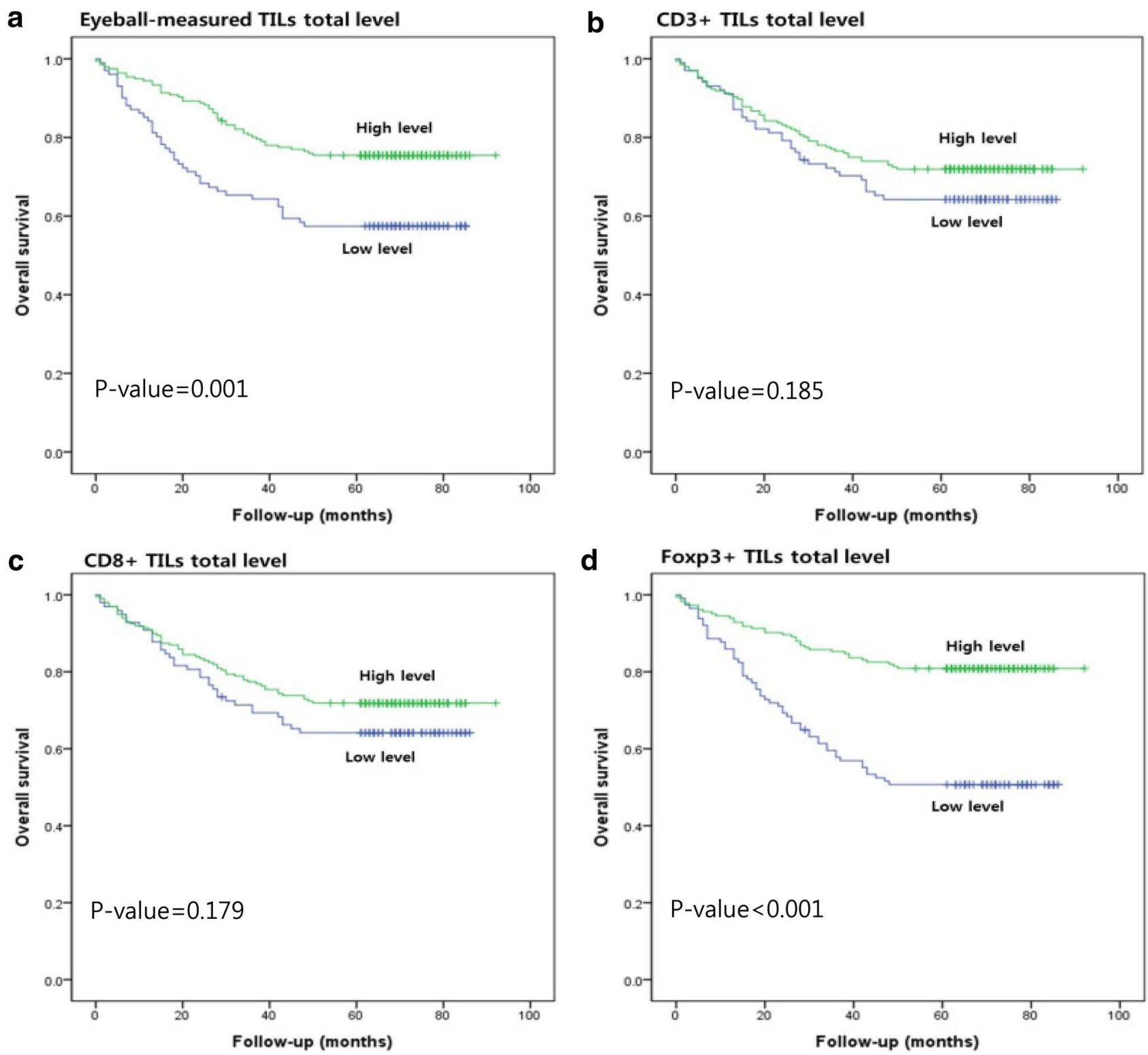

Fig. 1 Overall survival according to the immune cell type in immune contexture. Kaplan-Meier survival curves for overall survival based on TIL level in tumor center and invasive margin; a visual estimate of TILs, b CD $3^{+}$TILs, $\mathbf{c}$ CD $8^{+}$TILs, $\mathbf{d}$ Foxp $3^{+}$TILs

\section{Discussion}

This study investigated the immune contexture including type, density, and location of TILs, PD-L1 on tumor cells, and TILs in accordance with the molecular subtypes of gastric cancer. The density of each TIL differed significantly in accordance with the molecular subtype. $\mathrm{CD}^{+}, \mathrm{CD} 8^{+}$, and Foxp $3^{+}$immune cells displayed the greatest density in subtypes $\mathrm{C} 1$ and $\mathrm{C} 2$, whereas the $\mathrm{C} 3$ and $\mathrm{C} 4$ subtypes showed a low density of all types of immune cells. Type II tumors (low level of TILs/low PD-L1 expression levels) were significantly correlated with poor overall survival and accounted for the greatest proportion in gastric cancers aberrantly expressing p53 (subtype C4). These findings partly reveal the differences in the tumor microenvironment based on the molecular subtype of gastric cancer, thus revealing their potential therapeutic relevance.

With respect to immune contextures in gastric cancer, high levels of visually estimated TILs and Foxp $3^{+}$TILs were markedly associated with better overall survival, concurrent with previous reports [14-17]. TILs markedly contribute to the immune response against tumors. Several studies have reported an association between TILs and clinical outcomes in patients with various types of cancer [15-17]. Regarding gastric cancer, certain studies have reported that a high TIL density in gastric cancer is associated with favorable 
Table 2 The relationship between PD-L1 expression and clinicopathologic characteristics of gastric cancer

\begin{tabular}{|c|c|c|c|c|c|c|}
\hline & \multicolumn{3}{|c|}{ PD-L1 expression on tumor cells } & \multicolumn{3}{|c|}{ PD-L1 expression on TILs } \\
\hline & Negative & Positive & $P$ value & Negative & Positive & $P$ value \\
\hline Number (\%) & 279 (93.9) & $18(6.1)$ & & 273 (91.9) & $24(8.1)$ & \\
\hline Age & & & 0.120 & & & 0.531 \\
\hline Mean \pm SD & $62.13 \pm 10.932$ & $66.33 \pm 13.266$ & & $62.26 \pm 11.019$ & $63.75 \pm 12.231$ & \\
\hline $\operatorname{Sex}$ & & & 0.391 & & & 0.038 \\
\hline Male & $190(68.1)$ & $14(77.8)$ & & $183(67.0)$ & $21(87.5)$ & \\
\hline Female & $89(31.9)$ & $4(22.2)$ & & $90(33.0)$ & $3(12.5)$ & \\
\hline Size $(m m)$ & & & 0.024 & & & 0.482 \\
\hline Mean \pm SD & $54.11 \pm 30.703$ & $71.22 \pm 36.127$ & & $54.77 \pm 31.046$ & $59.46 \pm 33.974$ & \\
\hline Location & & & 0.462 & & & 0.729 \\
\hline Cardia, EGJ & $14(5.0)$ & $1(5.6)$ & & $13(4.8)$ & $2(8.3)$ & \\
\hline Body & $123(44.1)$ & $11(61.1)$ & & $124(45.4)$ & $10(41.7)$ & \\
\hline Antrum, pylorus & $134(48.0)$ & $6(33.3)$ & & $128(46.9)$ & $12(50.0)$ & \\
\hline N/A & $8(2.9)$ & $0(0.0)$ & & $8(2.9)$ & $0(0.0)$ & \\
\hline Gross type & & & 0.259 & & & 0.152 \\
\hline Non-excavated & $208(74.6)$ & $16(88.9)$ & & $203(74.4)$ & $21(87.5)$ & \\
\hline Excavated & $71(25.4)$ & $2(11.1)$ & & $70(25.6)$ & $3(12.5)$ & \\
\hline Grade \& WHO classification & & & 0.902 & & & 0.156 \\
\hline W/D, M/D & $139(49.8)$ & $10(55.6)$ & & $133(48.7)$ & $16(66.7)$ & \\
\hline $\mathrm{P} / \mathrm{D}$ & $97(34.8)$ & $7(38.9)$ & & $96(35.2)$ & $8(33.3)$ & \\
\hline Signet ring cell & $35(12.5)$ & $1(5.6)$ & & $36(13.2)$ & $0(0.0)$ & \\
\hline Mucinous & $8(2.9)$ & $0(0.0)$ & & $8(2.9)$ & $0(0.0)$ & \\
\hline Lauren type & & & 0.783 & & & 0.032 \\
\hline Intestinal & $123(44.1)$ & $7(38.9)$ & & $119(43.6)$ & $11(45.8)$ & \\
\hline Diffuse & $111(39.8)$ & $7(38.9)$ & & $113(41.4)$ & $5(20.8)$ & \\
\hline Mixed & $45(16.1)$ & $4(22.2)$ & & $41(15.0)$ & $8(33.3)$ & \\
\hline pT stage & & & 0.043 & & & 0.229 \\
\hline $\mathrm{T} 1$ & $71(25.4)$ & $1(5.6)$ & & $68(24.9)$ & $4(16.7)$ & \\
\hline $\mathrm{T} 2$ & $53(19.0)$ & $3(16.7)$ & & $50(18.3)$ & $6(25.0)$ & \\
\hline T3 & $52(18.6)$ & $8(44.4)$ & & $52(19.0)$ & $8(33.3)$ & \\
\hline $\mathrm{T} 4$ & $103(36.9)$ & $6(33.3)$ & & $103(37.7)$ & $6(25.0)$ & \\
\hline pN stage & & & 0.852 & & & 0.080 \\
\hline NO & $114(40.9)$ & $6(33.3)$ & & $110(40.3)$ & $10(41.7)$ & \\
\hline $\mathrm{N} 1$ & $49(17.6)$ & $3(16.7)$ & & $44(16.1)$ & $8(33.3)$ & \\
\hline $\mathrm{N} 2$ & $46(16.5)$ & $4(22.2)$ & & $46(16.8)$ & $4(16.7)$ & \\
\hline $\mathrm{N} 3$ & $70(25.1)$ & $5(27.8)$ & & $73(26.7)$ & $2(8.3)$ & \\
\hline Lymphovascular invasion & & & 0.029 & & & 0.894 \\
\hline Positive & $136(48.7)$ & $4(22.2)$ & & $129(47.3)$ & $11(45.8)$ & \\
\hline Negative & $143(51.3)$ & $14(77.8)$ & & $144(52.7)$ & $13(54.2)$ & \\
\hline Perineural invasion & & & 0.715 & & & 0.989 \\
\hline Positive & $174(62.4)$ & $12(66.7)$ & & $171(62.6)$ & $15(62.5)$ & \\
\hline Negative & $105(37.6)$ & $6(33.3)$ & & $102(37.4)$ & $9(37.5)$ & \\
\hline$M S I$ & & & 0.033 & & & 0.032 \\
\hline MSI-H & $27(9.7)$ & $5(27.8)$ & & $26(9.5)$ & $6(25.0)$ & \\
\hline MSI-I/L & $252(90.3)$ & $13(72.2)$ & & $24.7(90.5)$ & $18(75.0)$ & \\
\hline
\end{tabular}

$S D$ indicates standard deviation, $E G J$ esophagogastric junction, $N / A$ not available, $W / D$ we differentiated tubular adenocarcinoma, $M / D$ moderately differentiated tubular adenocarcinoma, $P / D$ poorly differentiated tubular adenocarcinoma, $M S I$ microsatellite instability, $E B V$ Epstein-Barr virus, $E M T$ epithelial-mesenchymal transition 

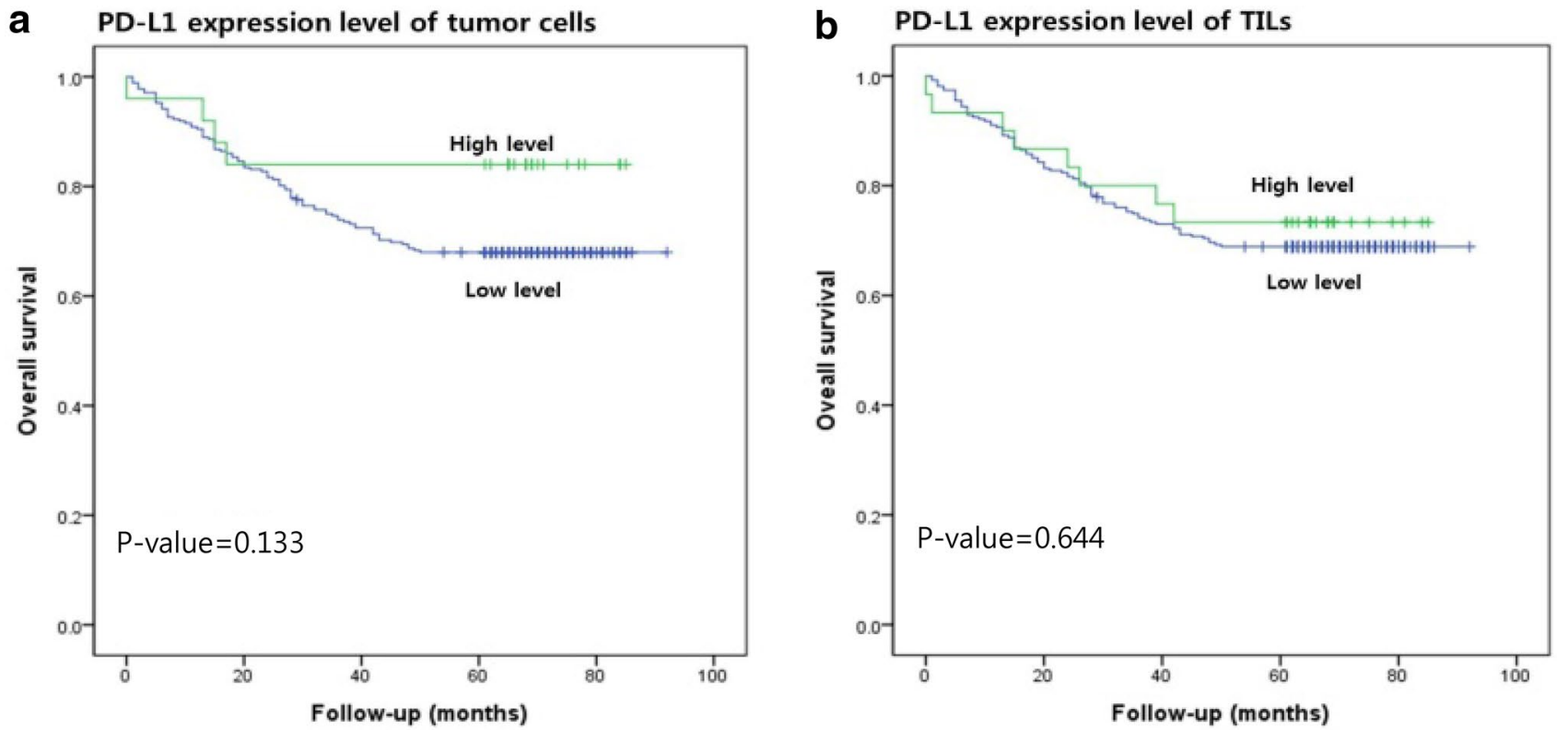

Fig. 2 Overall survival in the cohort according to the PD-L1 expression of tumor cells and TILs in tumor center and invasive margin. KaplanMeier survival curves for overall survival following total PD-L1 expression on tumor cells (a) and TILs (b)

outcomes, especially EBV-associated gastric cancer [18, 19]. Among TILs, we individually enumerated $\mathrm{CD} 3^{+}, \mathrm{CD} 8^{+}$, and Foxp $3^{+} \mathrm{T}$ cells and determined the absolute numbers of labeled TILs at the TC and IM of tumors. Lee et al. reported that $\mathrm{CD}^{+}$and $\mathrm{CD} 8^{+}$TILs are independent favorable prognostic factors in gastric cancer [18], as opposed to the present findings revealing no association with prognosis. Furthermore, the Foxp $3^{+} \mathrm{T}$ cell density was associated with better prognosis of gastric cancer in our datasets. Foxp3 is the transcription factor for regulatory $\mathrm{T}$ (Treg) cells, which are physiologically present in the immune system and maintain self-tolerance [20]. The clinical significance of Foxp $3^{+}$ $\mathrm{T}$ cells remains controversial among various cancer types. Regarding prognostic significance of Foxp $3^{+}$TILs in gastric cancer, different studies have reported conflicting results [21], probably because they comprise a different subpopulation of Foxp $3^{+} \mathrm{T}$ cells, potentially including a higher proportion of non-Treg cells [22]. Therefore, further studies are required to evaluate not only the absolute number of Foxp $3^{+}$ $\mathrm{T}$ cells, but also the components of Foxp $3^{+}$subpopulations in gastric cancers.

PD-L1 expression in gastric cancer was not associated with the prognosis of gastric cancer. Diverse interpretations of PD-L1 expression have been previously reported [23-27]. In the current study, we assessed PD-L1 expression levels on tumor cells and TILs and separately estimated the intensity and proportion of labeled cells. The prognostic significance of PD-L1 expression is controversial in not only gastric cancer, but also other malignancies [22-26]. Furthermore, immune subtypes (I-subtypes) based on the total
TIL levels and the PD-L1 expression levels in tumor cells, consistent with Teng's classification [28], were associated with patient prognosis, i.e., type II tumors (low total TILs and low PD-L1 status) were significantly correlated with poor overall survival.

We evaluated differences in immune contexture and PD-L1 expression based on the molecular subtype of gastric cancer. Subtypes C1 (EBV) and C2 (MSI), being subtypes with better prognosis, were significantly associated with high levels of immune density, including $\mathrm{CD}^{+}, \mathrm{CD}^{+}$, and Foxp $3^{+}$TILs and PD-L1 upregulation. The proportions of visually estimated and Foxp $3^{+}$TILs in the $\mathrm{C} 3$ subtype (aberrant E-cadherin expression; EMT) were lower than those in other subtypes. Carcinogenesis induced by microbe-associated inflammation could be prevented by tumor-infiltrating Foxp $3^{+}$Tregs, since Foxp $3^{+}$Tregs suppress the immune function of cytotoxic T cells induced by Helicobacter pylori. $[29,30]$. Thus, these results indicate low Foxp3 ${ }^{+}$TIL levels in diffuse-type gastric cancer (C3, aberrant E-cadherin expression) having less association with $\mathrm{H}$. pylori. Moreover, the results in this study suggest the prognostic or predictive implication of Foxp $3^{+}$TILs related to molecular subtype of gastric cancer.

Regarding the potential therapeutic relevance of gastric cancer, we assume that six cases (27.3\%) of gastric cancer of the $\mathrm{C} 1$ subtype also included type I tumors related to inflammatory signaling during adaptive immune resistance mechanisms. Since these tumors contain preexisting TILs, which were turned off via PD-L1, this group may comprise candidates for single-agent anti-PD-1/L1 blockade. Regarding the 
Table 3 Clinicopathologic characteristics according to the immune subtype (I-subtype)

\begin{tabular}{|c|c|c|c|c|c|}
\hline & Type I & Type II & Type III & Type IV & $P$ value \\
\hline Age & & & & & 0.065 \\
\hline Mean \pm SD & $66.24 \pm 13.723$ & $62.69 \pm 11.047$ & $69.63 \pm 7.501$ & $61.54 \pm 10.880$ & \\
\hline Sex & & & & & 0.452 \\
\hline Male & $10(58.8)$ & $69(74.2)$ & $6(75.0)$ & $119(66.5)$ & \\
\hline Female & $7(41.2)$ & $24(25.8)$ & $2(25.0)$ & $60(33.5)$ & \\
\hline Size $(\mathrm{mm})$ & & & & & 0.013 \\
\hline Mean & $58.29 \pm 31.096$ & $59.08 \pm 31.270$ & $83.88 \pm 35.126$ & $51.53 \pm 30.408$ & \\
\hline Location & & & & & 0.430 \\
\hline Cardia, EGJ & $1(5.9)$ & $4(4.3)$ & $0(0.0)$ & $10(5.6)$ & \\
\hline Body & $9(52.9)$ & $33(35.5)$ & $3(37.5)$ & $89(49.7)$ & \\
\hline Antrum, pylorus & $7(41.2)$ & $54(58.1)$ & $5(62.5)$ & $74(41.3)$ & \\
\hline N/A & $0(0.0)$ & $2(2.2)$ & $0(0.0)$ & $6(3.4)$ & \\
\hline Gross type & & & & & 0.000 \\
\hline Non-excavated & $15(88.2)$ & $82(88.2)$ & $7(87.5)$ & $120(67.0)$ & \\
\hline Excavated & $2(11.8)$ & $11(11.8)$ & $1(12.5)$ & $59(33.0)$ & \\
\hline Grade \& WHO classification & & & & & 0.094 \\
\hline W/D, M/D & $9(52.9)$ & $55(59.1)$ & $4(50.0)$ & $81(45.3)$ & \\
\hline $\mathrm{P} / \mathrm{D}$ & $8(47.1)$ & $29(31.2)$ & $3(37.5)$ & $64(35.8)$ & \\
\hline Signet ring cell & $0(0.0)$ & $5(5.4)$ & $1(12.5)$ & $30(16.8)$ & \\
\hline Mucinous & $0(0.0)$ & $4(4.3)$ & $0(0.0)$ & $4(2.2)$ & \\
\hline Lauren type & & & & & 0.178 \\
\hline Intestinal & $6(35.3)$ & $51(54.8)$ & $3(37.5)$ & $70(39.1)$ & \\
\hline Diffuse & $6(35.3)$ & $30(32.3)$ & $4(50.0)$ & $78(43.6)$ & \\
\hline Mixed & $5(29.4)$ & $12(12.9)$ & $1(12.5)$ & $31(17.3)$ & \\
\hline pT stage & & & & & 0.000 \\
\hline $\mathrm{T} 1$ & $2(11.8)$ & $6(6.5)$ & $0(0.0)$ & $64(35.8)$ & \\
\hline $\mathrm{T} 2$ & $6(35.3)$ & $19(20.4)$ & $0(0.0)$ & $31(17.3)$ & \\
\hline $\mathrm{T} 3$ & $5(29.4)$ & $20(21.5)$ & $3(37.5)$ & $32(17.9)$ & \\
\hline $\mathrm{T} 4$ & $4(23.5)$ & $48(51.6)$ & $5(62.5)$ & $52(29.1)$ & \\
\hline pN stage & & & & & 0.000 \\
\hline No & $7(41.2)$ & $19(20.4)$ & $4(50.0)$ & $90(50.3)$ & \\
\hline N1 & $3(17.6)$ & $18(19.4)$ & $2(25.0)$ & $29(16.2)$ & \\
\hline $\mathrm{N} 2$ & $3(17.6)$ & $24(25.8)$ & $1(12.5)$ & $22(12.3)$ & \\
\hline N3 & $4(23.5)$ & $32(34.4)$ & $1(12.5)$ & $38(21.2)$ & \\
\hline Lymphovascular invasion & & & & & 0.000 \\
\hline Positive & $5(29.4)$ & $60(64.5)$ & $2(25.0)$ & $73(40.8)$ & \\
\hline Negative & $12(70.6)$ & $33(35.5)$ & $6(75.0)$ & $106(59.2)$ & \\
\hline Perineural invasion & & & & & 0.000 \\
\hline Positive & $7(41.2)$ & $72(77.4)$ & $8(100.0)$ & $99(55.3)$ & \\
\hline Negative & $10(58.8)$ & $21(22.6)$ & $0(0.0)$ & $80(44.7)$ & \\
\hline$M S I$ & & & & & 0.000 \\
\hline MSI-H & $6(35.3)$ & $5(5.4)$ & $4(50.0)$ & $17(9.5)$ & \\
\hline MSI-I/L & $11(64.7)$ & $88(94.6)$ & $4(50.0)$ & $162(90.5)$ & \\
\hline
\end{tabular}

$S D$ indicates standard deviation, $E G J$ esophagogastric junction, $N / A$ not available, $W / D$ we differentiated tubular adenocarcinoma, $M / D$ moderately differentiated tubular adenocarcinoma, $P / D$ poorly differentiated tubular adenocarcinoma, $M S I$ microsatellite instability
C4 (aberrant p53) subtype, 51 cases (42.9\%) also included type II tumors showing a lack of detectable immune reactions-so-called immunologic ignorance. However, single-agent checkpoint blockade would be ineffective in this group. Alternatively, combinatorial therapy comprising an agent to generate an immunogenic tumor environment and 


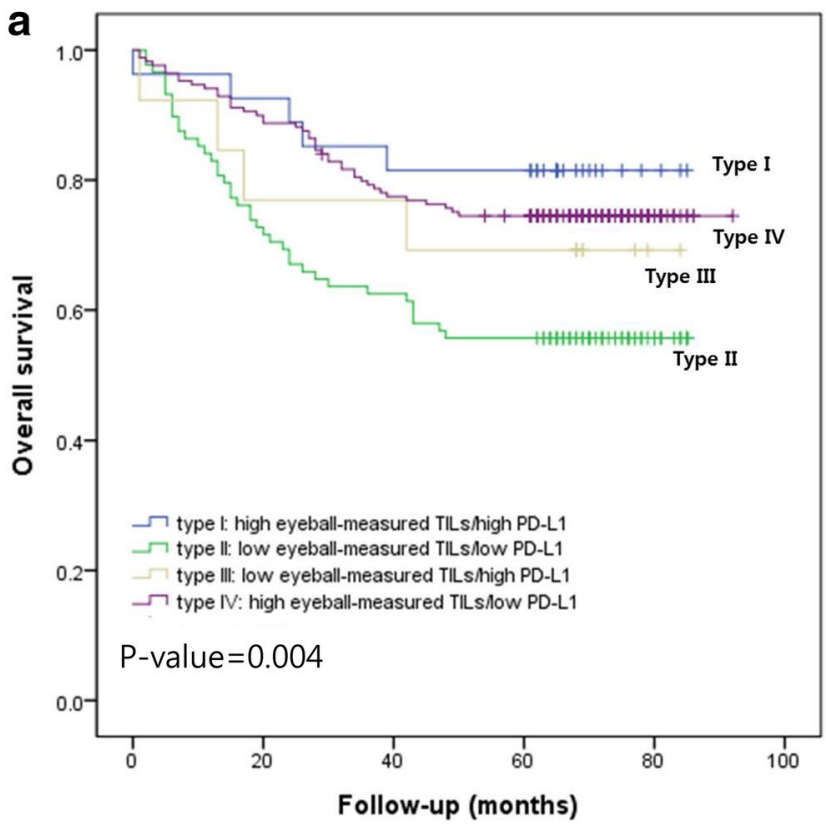

Fig. 3 Overall survival according to immune subtype and molecular subtype. Kaplan-Meier survival curves for overall survival following as immune subtype (I-subtype) based on the level of TILs and the sta-

an immune checkpoint agent could be considered. On using a combination of anti-CTLA-4 and anti-PD-1 antibodies, CTLA-4 blockade induces tumor infiltration by activated T cells and a concomitant increase in IFN- $\gamma$. Finally, PD-L1 was induced in the tumor environment [31]. In this context, $\mathrm{C} 4$ with aberrant $\mathrm{p} 53$ expression can show improved clinical outcomes through combinatorial therapy. A recent phase $1 \mathrm{~b}$ clinical trial of the anti-PD-1 antibody pembrolizumab in gastric cancer demonstrated significant antitumor effects [31]. More than $60 \%$ of C3 (EMT) and C5 (normal p53) gastric cancers showed a type IV (immune tolerance) tumor microenvironment (high TILs level and low PD-L1 status). However, although type IV tumors contain high levels of TILs, they comprise a heterogeneous population of lymphoid cells. Moreover, type IV tumors may undergo another suppressive pathway, rather than the PD-1/PD-L1 immune checkpoint pathway. With respect to type IV tumors, immunotherapeutic strategies are yet incomplete. Therefore, appropriate selection of patients potentially benefiting from immunotherapy is important, and a consensus regarding the immunohistochemical determination of TILs level and PD-L1 is mandatory.

In conclusion, this study shows that the tumor microenvironment including immune contextures and PD-L1 expression levels differs depending on the molecular subtypes, and these immunologic signatures are significantly associated with overall survival. Differences in the tumor microenvironment account for differences in tumor development and progression in accordance with the molecular subtypes and

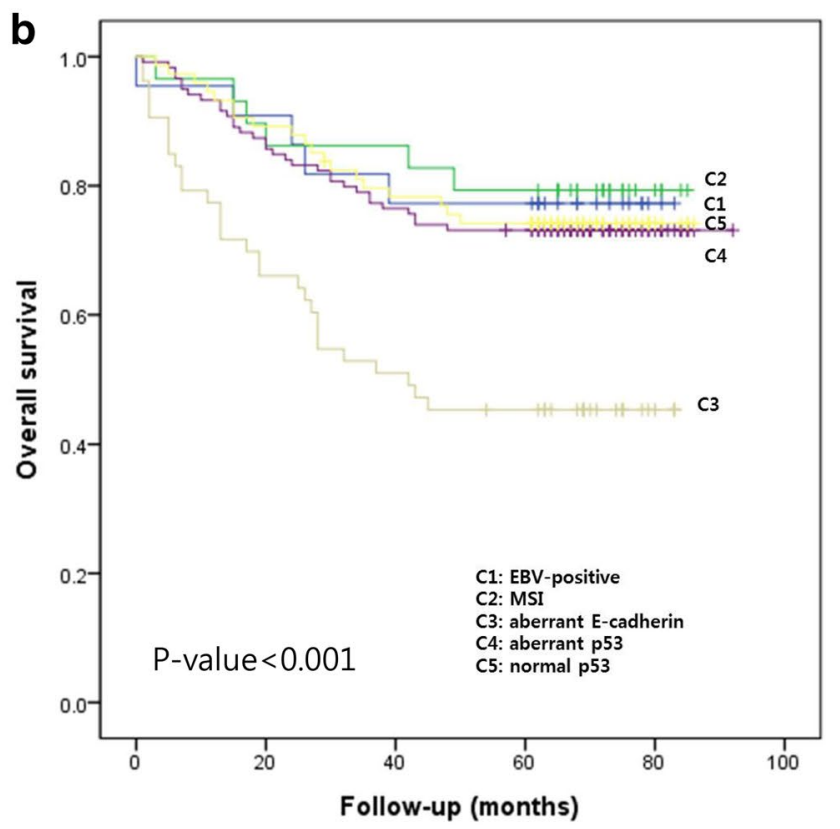

tus of PD-L1 (a) and the molecular subtype (C-subtype) (b). $P$ value was calculated from log-rank test

can also provide additional treatment alternatives including immunotherapy. Ultimately, an understanding of the molecular and immunological characteristics in gastric cancers is expected to contribute to improved patient survival. Therefore, evaluation of the immune contexture and a consistent interpretation of PD-L1 expression status are essential to select an appropriate immunotherapeutic candidate.

Acknowledgements This work was supported by the National Research Foundation of Korea (NRF) Grant funded by the Korea government (MSIT) (No. NRF-2018R1C1B6004754). The biospecimens for this study were provided by the Pusan National University Hospital, members of the National Biobank of Korea, which is supported by the Ministry of Health, Welfare, and Family Affairs.

\section{Compliance with ethical standards}

Conflict of interest The authors declare that they have no association with or financial interest in any commercial company relevant to this study.

Ethical approval This article does not contain any studies with human participants or animals performed by any of the authors.

\section{References}

1. Hanahan D, Weinberg RA. Hallmarks of cancer: the next generation. Cell. 2011;144:646-74.

2. Cancer Genome Atlas Research Network. Comprehensive molecular characterization of gastric adenocarcinoma. Nature. 2014;513:202-9. 
Table 4 Immune contexture and PD-L1 expression based on the molecular subtype of gastric cancer (C-subtype)

\begin{tabular}{|c|c|c|c|c|c|c|}
\hline & C1 (EBV) & C2 (MSI) & C3 (EMT) & C4 (aberrant p53) & C5 (normal p53) & $P$ value \\
\hline Number (\%) & $22(7.4)$ & $29(9.8)$ & $53(17.8)$ & $119(40.1)$ & $74(24.9)$ & \\
\hline \multicolumn{7}{|l|}{ [Tumor center] } \\
\hline Visual estimate of TIL level & & & & & & 0.000 \\
\hline Low & $0(0.0)$ & $15(51.7)$ & $27(50.9)$ & $74(62.2)$ & $35(47.3)$ & \\
\hline High & $22(100.0)$ & $14(48.3)$ & $26(49.1)$ & $45(37.8)$ & $39(52.7)$ & \\
\hline CD3 level & & & & & & 0.001 \\
\hline Low & $3(13.6)$ & $11(37.9)$ & $30(56.6)$ & $71(59.7)$ & $35(47.3)$ & \\
\hline High & $19(86.4)$ & $18(62.1)$ & $23(43.4)$ & $48(40.3)$ & $39(52.7)$ & \\
\hline CD8 level & & & & & & 0.000 \\
\hline Low & $2(9.1)$ & $10(34.5)$ & $30(56.6)$ & $67(56.3)$ & $40(54.1)$ & \\
\hline High & $20(90.9)$ & $19(65.5)$ & $23(43.4)$ & $52(43.7)$ & $34(45.9)$ & \\
\hline Foxp3 level & & & & & & 0.005 \\
\hline Low & $4(18.2)$ & $12(41.4)$ & $32(60.4)$ & $69(58.0)$ & $37(50.0)$ & \\
\hline High & $18(81.8)$ & 17 (58.6) & $21(39.6)$ & $50(42.0)$ & $37(50.0)$ & \\
\hline PD-L1 of tumor cells & & & & & & 0.007 \\
\hline Negative & $17(77.3)$ & $25(86.2)$ & $51(96.2)$ & 115 (96.6) & $71(95.9)$ & \\
\hline Positive & $5(22.7)$ & $4(13.8)$ & $2(3.8)$ & $4(3.4)$ & $3(4.1)$ & \\
\hline$P D-L 1$ of TILs & & & & & & 0.001 \\
\hline Negative & $15(68.2)$ & $24(82.8)$ & $51(96.2)$ & $112(94.1)$ & $71(95.9)$ & \\
\hline Positive & $7(31.8)$ & $5(17.2)$ & $2(3.8)$ & $7(5.9)$ & $3(4.1)$ & \\
\hline \multicolumn{7}{|l|}{ [Invasive margin] } \\
\hline Visual estimate of TIL level & & & & & & 0.016 \\
\hline Low & $4(18.2)$ & $14(48.3)$ & $25(47.2)$ & $69(58.0)$ & $38(51.4)$ & \\
\hline High & $18(81.8)$ & $15(51.7)$ & $28(52.8)$ & $50(42.0)$ & $36(48.6)$ & \\
\hline CD3 level & & & & & & 0.018 \\
\hline Low & $5(22.7)$ & $10(34.5)$ & $28(52.8)$ & $67(56.3)$ & $40(54.1)$ & \\
\hline High & $17(77.3)$ & $19(65.5)$ & $25(47.2)$ & $52(43.7)$ & $34(45.9)$ & \\
\hline CD8 level & & & & & & 0.001 \\
\hline Low & $3(13.6)$ & $10(34.5)$ & $31(58.5)$ & $64(53.8)$ & $44(59.5)$ & \\
\hline High & $19(86.4)$ & $19(65.5)$ & $22(41.5)$ & $55(46.2)$ & $30(40.5)$ & \\
\hline Foxp3 level & & & & & & 0.000 \\
\hline Low & $2(9.1)$ & $10(34.5)$ & $36(67.9)$ & $68(57.1)$ & $39(52.7)$ & \\
\hline High & $20(90.9)$ & $19(65.5)$ & $17(32.1)$ & $51(42.9)$ & $35(47.3)$ & \\
\hline PD-L1 of tumor cells & & & & & & 0.025 \\
\hline Negative & $18(81.8)$ & $25(86.2)$ & $51(96.2)$ & 115 (96.6) & $71(95.9)$ & \\
\hline Positive & $4(18.2)$ & $4(13.8)$ & $2(3.8)$ & $4(3.4)$ & $3(4.1)$ & \\
\hline$P D-L 1$ of $T I L s$ & & & & & & 0.000 \\
\hline Negative & $16(72.7)$ & $24(82.8)$ & $51(96.2)$ & $117(98.3)$ & $73(98.6)$ & \\
\hline Positive & $6(27.3)$ & $5(17.2)$ & $2(3.8)$ & $2(1.7)$ & $1(1.4)$ & \\
\hline \multicolumn{7}{|l|}{ [PD-L1 level] } \\
\hline PD-L1 level of tumor cells & & & & & & 0.000 \\
\hline Low & $16(72.7)$ & $22(75.9)$ & $50(94.3)$ & $114(95.8)$ & $70(94.6)$ & \\
\hline High & $6(27.3)$ & $7(24.1)$ & $3(5.7)$ & $5(4.2)$ & $4(5.4)$ & \\
\hline PD-L1 level of TILs & & & & & & 0.000 \\
\hline Low & $13(59.1)$ & $21(72.4)$ & $51(96.2)$ & $112(94.1)$ & $70(94.6)$ & \\
\hline High & $9(40.9)$ & $8(27.6)$ & $2(3.8)$ & $7(5.9)$ & $4(5.4)$ & \\
\hline [Total PD-L1 status] & & & & & & 0.000 \\
\hline Low & $11(50.0)$ & $19(65.5)$ & $50(94.3)$ & $108(90.8)$ & $69(93.2)$ & \\
\hline High & $11(50.0)$ & $10(34.5)$ & $3(5.7)$ & $11(9.2)$ & $5(6.8)$ & \\
\hline [I subtype] & & & & & & N/A \\
\hline Type I (high TILs/high PD-L1) & $6(27.3)$ & $5(17.2)$ & $0(0.0)$ & $3(2.5)$ & $3(4.1)$ & \\
\hline Type II (low TILs/low PD-L1) & $0(0.0)$ & $6(20.7)$ & $14(26.4)$ & $51(42.9)$ & $22(29.7)$ & \\
\hline Type III (low TILs/high PD-L1) & $0(0.0)$ & $2(6.9)$ & $3(5.7)$ & $2(1.7)$ & $1(1.4)$ & \\
\hline Type IV (high TILs/low PD-L1) & $16(72.7)$ & $16(55.2)$ & $36(67.9)$ & $63(52.9)$ & $48(64.9)$ & \\
\hline
\end{tabular}


Table 4 (continued)

$E B V$ indicates Epstein-Barr virus, $M S I$ microsatellite instability, EMT epithelial-mesenchymal transition, TILs tumor-infiltrating lymphocytes,

Foxp3 forkhead box protein 3, $P D-L 1$ programmed death-ligand 1

3. Setia N, Agoston AT, Han HS, Mullen JT, Duda DG, Clark JW, et al. A protein and mRNA expression-based classification of gastric cancer. Mod Pathol. 2016;29:772-84.

4. Ahn S, Lee SJ, Kim Y, Kim A, Shin N, Choi KU, et al. Highthroughput protein and mRNA expression-based classification of gastric cancers can identify clinically distinct subtypes, concordant with recent molecular classifications. Am J Surg Pathol. 2017;41:106-15.

5. Ogino S, Galon J, Fuchs CS, Dranoff G. Cancer immunologyanalysis of host and tumor factors for personalized medicine. Nat Rev Clin Oncol. 2011;8:711-9.

6. Pardoll DM. The blockade of immune checkpoints in cancer immunotherapy. Nat Rev Cancer. 2012;12:252-64.

7. Wu C, Zhu Y, Jiang J, Zhao J, Zhang XG, Xu N. Immunohistochemical localization of programmed death-1 ligand-1 (PD-L1) in gastric carcinoma and its clinical significance. Acta Histochem. 2006;108:19-24.

8. Hou J, Yu Z, Xiang R, Li C, Wnag L, Chen S, Li Q, et al. Correlation between infiltration of $\mathrm{FOXP}^{+}$regulatory $\mathrm{T}$ cells and expression of B7-H1 in the tumor tissues of gastric cancer. Exp Mol Pathol. 2014;96:284-91.

9. Moehler MH, Cho JY, Kim YH, Kim JW, Bartolomeo MD, Ajani JA, et al. A randomized, open-label, two-arm phase II trial comparing the efficacy of sequential ipilimumab versus best supportive care (BSC) following first line chemotherapy in patients with unresectable, locally advanced/metastic gastric or gastroesophageal junction cancer. ASCO Meet Abstr. 2016;34:4011.

10. Fuchs CS, Doi T, Jang RW, Muro K, Satoh T, Machado M, et al. KEYNOTE-059 cohort 1: efficacy and safety of pembrolizumab (pembro) monotherapy in patients with previously treated advanced gastric cancer. J Clin Oncol. 2017:35:4003.

11. Wainberg ZA, Jalal S, Muro K, Yoon HH, Garrido M, Golan T, et al. LBA28 PRKEYNOTE-059 update: efficacy and safety of pembrolizumab alone or in combination with chemotherapy in patients with advanced gastric or gastroesophageal (G/GEJ) cancer. Ann Oncol. 2017;28:v605-49.

12. Bang YJ, Muro K, Fuchs CS, Golan T, Geva R, Hara H, et al. KEYNOTE-059 cohort2: safety and efficacy of pembrolizumab (pembro) plus 5-fluorouracil (5-FU) and cisplatin for firstline (1L) treatment of advanced gastric cancer. J Clin Oncol. 2017;35:4012.

13. Li Z, Lai Y, Sun L, Zhang X, Liu R, Feng G, et al. PD-L1 expression is associated with massive lymphocyte infiltration and histology in gastric cancer. Hum Pathol. 2016;55:182-9.

14. Fridman WH, Pages F, Sautes-Fridman C, Galon J. The immune contexture in human tumours: impact on clinical outcome. Nat Rev Cancer. 2012;12:298-306.

15. Lipponen PK, Eskelinen MJ, Jauhiainen K, Harju E, Terho $\mathrm{R}$. Tumour infiltrating lymphocytes as an independent prognostic factor in transitional cell bladder cancer. Eur $\mathrm{J}$ Cancer. 1992;29A:69-75.

16. Karja V, Aaltomaa S, Lipponen P, Isotalo T, Talja M, Mokka R. Tumour-infiltrating lymphocytes: a prognostic factor of PSA-free survival in patients with local prostate carcinoma treated by radical prostatectomy. Anticancer Res. 2005;25:4435-8.

17. Ropponen KM, Eskelinen MJ, Lipponen PK, Alhava E, Kosma VM. Prognostic value of tumour-infiltrating lymphocytes (TILs) in colorectal cancer. J Pathol. 1997;182:318-24.
18. Lee HE, Chae SW, Lee YJ, Kim MA, Lee HS, Lee BL, et al. Prognostic implications of type and density of tumour-infiltrating lymphocytes in gastric cancer. Br J Cancer. 2008;99(10):1704-11.

19. Kang BW, Seo AN, Yoon S, Bae HI, Jeon SW, Kwon OK, et al. Prognostic value of tumor-infiltrating lymphocytes in Epstein-Barr virus-associated gastric cancer. Ann Oncol. 2016;27:494-501.

20. Wing K, Sakaguchi S. Regulatory $\mathrm{T}$ cells exert checks and balances on self tolerance and autoimmunity. Nat Immunol. 2010;11:7-13.

21. Shang B, Liu Y, Jiang SJ, Liu Y. Prognostic value of tumor-infiltrating FoxP $3^{+}$regulatory $\mathrm{T}$ cells in cancers: a systematic review and meta-analysis. Sci Rep. 2015;5:15179.

22. Miyara M, Yoshioka Y, Kitoh A, Shima T, Wing K, Niwa A, et al. Functional delineation and differentiation dynamics of human $\mathrm{CD} 4^{+} \mathrm{T}$ cells expressing the FoxP3 transcription factor. Immunity. 2009;30:899-911.

23. Boger C, Behrens HM, Mathiak M, Kruger S, Kalthoff H, Rocken C. PD-L1 is an independent prognostic predictor in gastric cancer of Western patients. Oncotarget. 2016;7:24269-83.

24. Saito R, Abe H, Kunita A, Yamashita H, Seto Y, Fukayama M. Overexpression and gene amplification of PD-L1 in cancer cells and PD-L1 ${ }^{+}$immune cells in Epstein-Barr virus-associated gastric cancer: the prognostic implications. Mod Pathol. 2017;30:427-39.

25. Li Z, Lai Y, Sun L, Zhang X, Liu R, Feng G, et al. PD-L1 expression is associated with massive lymphocyte infiltration and histology in gastric cancer. Hum Pathol. 2016;55:182-9.

26. Dong M, Wang HY, Zhao XX, Chen JN, Zhang YW, Huang Y, et al. Expression and prognostic roles of PIK3CA, JAK2, PD-L1, and PD-L2 in Epstein-Barr virus-associated gastric carcinoma. Hum Pathol. 2016;53:25-34.

27. Chang H, Jung WY, Kang Y, Lee H, Kim A, Kim HK, et al. Programmed death-ligand 1 expression in gastric adenocarcinoma is a poor prognostic factor in a high $\mathrm{CD}^{+}$tumor infiltrating lymphocytes group. Oncotarget. 2016;7:80426-34.

28. Teng MW, Ngiow SF, Ribas A, Smyth MJ. Classifying cancers based on T-cell infiltration and PD-L1. Cancer Res. 2015;75:2139-45.

29. Shang B, Liu Y, Jiang SJ, Liu Y. Prognostic value of tumor-infiltrating $\mathrm{FoxP}^{+}$regulatory $\mathrm{T}$ cells in cancers: a systematic review and meta-analysis. Sci Rep. 2015;5:15179.

30. Arnold IC, Lee JY, Amieva MR, Roers A, Flavell RA, Sparwasser $\mathrm{T}$, et al. Tolerance rather than immunity protects from Helicobacter pylori-induced gastric preneoplasia. Gastroenterology. 2011;140:199-209.

31. Huang RR, Jalil J, Economou JS, Chmielowski B, Koya RC, Mok $\mathrm{S}$, et al. CTLA4 blockade induces frequent tumor infiltration by activated lymphocytes regardless of clinical responses in humans. Clin Cancer Res. 2011;17:4101-9.

Publisher's Note Springer Nature remains neutral with regard to jurisdictional claims in published maps and institutional affiliations. 